\title{
GMR
}

\section{Correlation between polymorphisms of hypoxia-inducible factor-1 $\alpha$ Pro582Ser and type 2 diabetic nephropathy}

\author{
Y.X. Bi* , L. Yu* and G.X. Jin \\ Department of Endocrinology, the First Affiliated Hospital of Bengbu Medical College, \\ Bengbu, Anhui Province, China \\ *These authors contributed equally to this study. \\ Corresponding author: G.X. Jin \\ E-mail: jyzjyz1999@163.com \\ Genet. Mol. Res. 14 (4): 14503-14509 (2015) \\ Received June 19, 2015 \\ Accepted September 1, 2015 \\ Published November 18, 2015 \\ DOI http://dx.doi.org/10.4238/2015.November.18.13
}

ABSTRACT. We examined the correlation between gene polymorphisms in hypoxia-inducible factor-1a (HIF-1 $\alpha$ ) Pro582Ser and type 2 diabetic nephropathy (DN). A total of 244 subjects with type 2 diabetes were recruited. The 1285-bp locus polymorphism of HIF-1a exon was detected using polymerase chain reaction-restriction fragment length polymorphism. $\mathrm{C} / \mathrm{T}$ single nucleotide polymorphisms were detected at the site of 1285 bp of the HIF-1a exon, from a proline to a serine (Pro582Ser). The frequency of CT heterozygotes was significantly higher in DN patients than in diabetes patients $(P<0.05)$. Logistic regression analysis showed that high hemoglobin $\mathrm{A} 1 \mathrm{C}$ and low high-density lipoprotein-cholesterol were risk factors for DN, and Pro582Ser was excluded in the equation. HIF-1a Pro582Ser single nucleotide polymorphisms may be correlated with type $2 \mathrm{DN}$, which needs further exploration.

Key words: Diabetic nephropathy; Gene polymorphism; Hypoxia-inducible factor- $1 \alpha$ 


\section{INTRODUCTION}

Diabetic nephropathy (DN) is the most common microvascular complication of diabetes mellitus (DM); studies have shown that DN may be related to heredity and the environment. Hypoxia is an important factor in the pathogenesis of DN (Tanaka and Nangaku, 2010). Hypoxiainducible factor-1 (HIF-1) is an important regulator of cell oxygen metabolism and is composed of $\alpha$ and $\beta$ subunits; its activity is mainly dependent on the strict control of HIF-1 $\alpha$ degradation. HIF$1 \alpha$ is a transcriptional activator that regulates gene expression by changing intracellular oxygen concentrations, allowing cells to adapt to hypoxia conditions by regulating the expression of related downstream genes (Semenza, 2010). Previous studies have shown that the HIF-1a Pro582Ser polymorphism (rs11549465) was correlated with type 1 DN (Gu et al., 2013), but its relationship with type $2 \mathrm{DN}$ remains unclear. Therefore, we examined the correlation between the HIF-1a Pro582Ser polymorphism and type 2 DN.

\section{MATERIAL AND METHODS}

\section{Subjects}

According to diagnosis and classification standards of diabetes proposed by the WHO in 1999, 244 cases of type 2 diabetes were selected. Based on urinary albumin excretion rates, grouping was performed: 104 cases showed urinary albumin excretion rates $<20 \mathrm{mg} / \mathrm{min}$ and were included in the DM group; 140 cases showed urinary albumin excretion rates $>20 \mathrm{mg} / \mathrm{min}$ or $24-\mathrm{h}$ urinary protein $>0.5 \mathrm{~g}$ and were included in the DN group. Exclusion criteria were as follows: proteinuria caused by heart and liver, primary urinary system diseases, and the use of drugs affecting renal function.

\section{Methods}

\section{Clinical data}

Blood pressure, height, and weight were measured and body mass index was calculated; serum creatinine (SCr), blood urea nitrogen, fasting plasma glucose, triglycerides (TG), total cholesterol, low-density lipoprotein cholesterol, high-density lipoprotein-cholesterol (HDL-C), hemoglobin A1c (HbA1c), fasting C-peptide, 2-h postprandial plasma glucose, and 2-h postprandial C-peptide were detected. The 24-h urine specimens of patients were collected to detect microalbumin.

\section{Polymorphism detection}

A 5-mL peripheral blood sample was collected and the DNA of white blood cells was isolated using a DNA extraction kit. Primers of the target genes were as follows: upstream primer, 5'-AAGGTGTGGCCATTGTAAAAACTC-3'; downstream primer, 5'-GCACTAGTAGTTTCTTTATGT ATG-3'. The gene was amplified using polymerase chain reaction instrument; the length of target gene fragment was $346 \mathrm{bp}$. Restriction digestion of the target gene fragment was performed using $\mathrm{Hphl}$ endonuclease at $37^{\circ} \mathrm{C}$ in a water bath overnight, after which the samples were analyzed by electrophoresis. 


\section{Statistical analysis}

The SPSS 17.0 statistical software was used for data analysis (SPSS, Inc., Chicago, IL, USA). Measurement data showing a normal distribution are reported as means $\pm S D$, and differences between groups were compared using the independent sample $t$-test. Measurement data that did not show a normal distribution are reported as median (Q25, Q75), and the differences between the groups were compared using the Wilcoxon rank sum test. Hardy-Weinberg genetic equilibrium and differences in allele frequencies and gender composition between the DN group and non-DN group were analyzed using the $\chi^{2}$ test. Risk factors for DN were analyzed by logistic regression analysis. $\mathrm{P}<0.05$ indicated that the difference was statistically significant.

\section{RESULTS}

\section{Biochemical tests}

TG, SCr, and blood urea nitrogen were higher and HDL-C was lower in the DN group than in the DM group $(\mathrm{P}<0.05$; Table 1$)$.

Table 1. General characteristics and clinical data of DN and DM groups.

\begin{tabular}{lccc}
\hline & DN group $(\mathrm{N}=140)$ & DM group $(\mathrm{N}=104)$ & P value \\
\hline Gender (male/female) & $72 / 68$ & $62 / 42$ & 0.20 \\
Age (years) & $54.76 \pm 14.79$ & $54.63 \pm 14.92$ & 0.95 \\
SBP $(\mathrm{mmHg})$ & $135.58 \pm 20.87$ & $131.80 \pm 16.60$ & 0.13 \\
DBP $(\mathrm{mmHg})$ & $82.7 \pm 14.60$ & $82.9 \pm 14.03$ & 0.91 \\
BMI $\left(\mathrm{kg} / \mathrm{m}^{2}\right)$ & $23.17 \pm 3.41$ & $23.97 \pm 2.95$ & 0.06 \\
FPG $(\mathrm{mM})$ & $9.01 \pm 3.81$ & $8.65 \pm 3.86$ & 0.47 \\
LPG $(\mathrm{mM})$ & $16.13 \pm 8.85$ & $14.21 \pm 5.58$ & 0.06 \\
FCP $(\mathrm{ng} / \mathrm{mL})$ & $1.56 \pm 1.31$ & $1.35 \pm 1.16$ & 0.21 \\
LCP $(\mathrm{ng} / \mathrm{mL})$ & $4.02 \pm 3.39$ & $3.31 \pm 2.70$ & 0.08 \\
TG $(\mathrm{mM})$ & $2.12 \pm 2.50$ & $1.64 \pm 1.01$ & 0.04 \\
TC $(\mathrm{mM})$ & $4.74 \pm 1.69$ & $4.76 \pm 1.45$ & 0.92 \\
LDL-C $(\mathrm{mM})$ & $3.06 \pm 1.30$ & $2.87 \pm 1.25$ & 0.25 \\
HDL-C $(\mathrm{mM})$ & $1.06 \pm 0.34$ & $1.22 \pm 1.60$ & 0.01 \\
HbA1 $/ \%$ & $11.33 \pm 10.04$ & $9.67 \pm 2.44$ & 0.06 \\
BUN $(\mathrm{mM})$ & $9.47 \pm 8.64$ & $6.10 \pm 2.47$ & $<0.01$ \\
SCr $(\mu \mathrm{M})$ & $131.5 \pm 40.56$ & $69.51 \pm 28.70$ & $<.01$ \\
\hline
\end{tabular}

\section{Digestion results}

The CC genotype of rs11549465 contained $\mathrm{Hphl}$ restriction sites, resulting in 2 fragments of 228 and $118 \mathrm{bp}$. The heterozygous variant (CT) showed 3 segments of 346, 228, and $118 \mathrm{bp}$ (Figure 1). The homozygous variant (TT), because of the loss of restriction sites, showed a 346-bp fragment (this variant was not observed in this study).

\section{Hardy-Weinberg test}

There was no significant difference between the observed and predicted values of the rs11549465 genotype polymorphism both in the DN and DM groups $(P>0.05)$. The genotype distributions of the 2 groups were in Hardy-Weinberg equilibrium; the sample was representative (Table 2 ). 


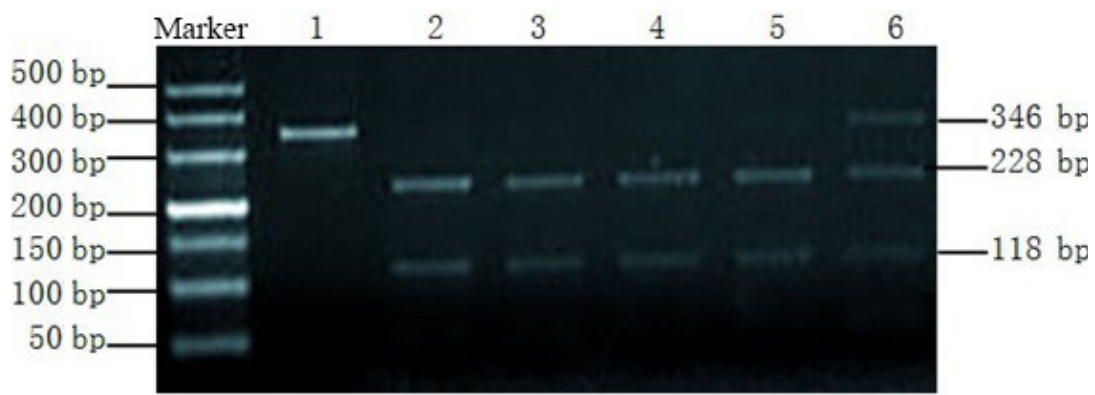

Figure 1. Restriction electrophoresis of target gene. Lane 1: undigested PCR product; lanes 2, 3, 4, and 5: CC genotype; lane 6: CT genotype.

\begin{tabular}{|c|c|c|c|c|c|c|c|c|c|}
\hline \multirow[t]{2}{*}{ Group } & \multirow[t]{2}{*}{ Case } & & \multicolumn{3}{|c|}{ Frequency } & \multicolumn{2}{|c|}{ Allele } & \multirow[t]{2}{*}{$\chi^{2}$} & \multirow[t]{2}{*}{$\mathrm{P}$} \\
\hline & & & $\mathrm{CC}$ & $\mathrm{CT}$ & $\mathrm{TT}$ & C & $\mathrm{T}$ & & \\
\hline \multirow[t]{2}{*}{$\mathrm{N}$ group } & 140 & Observed value & 130.00 & 10.0 & 0.0 & 270 & 10 & & \\
\hline & & Predicted value & 129.02 & 9.64 & 0.17 & & & 0.18 & $>0.05$ \\
\hline \multirow[t]{2}{*}{ M group } & 104 & Observed value & 88.0 & 16.0 & 0.0 & 192 & 16 & & \\
\hline & & Predicted value & 88.62 & 14.77 & 0.62 & & & 0.16 & $>0.05$ \\
\hline
\end{tabular}

\section{rs11549465 polymorphism: differences in T allele carrier rate between the DN group and the DM group}

In the DM group, the proportion of patients carrying the T allele was significantly higher than that in the DN group $(P<0.05)$. The odds ratio between the $T$ allele and $D N$ pathogenesis was 0.42 (Table 3).

Table 3. Comparison of T allele carrier rate between the 2 groups.

\begin{tabular}{|c|c|c|c|c|c|}
\hline \multirow[t]{2}{*}{ Group } & \multirow[t]{2}{*}{ Cases } & \multicolumn{2}{|c|}{ Genotype } & \multirow[t]{2}{*}{$P$ value } & \multirow[t]{2}{*}{ OR $(95 \% \mathrm{Cl})$} \\
\hline & & $\mathrm{CC}$ & CT & & \\
\hline DN group & 140 & $130(92.86 \%)$ & $10(7.14 \%)$ & & \\
\hline DM group & 104 & 88 (84.62\%) & $16(15.38 \%)$ & 0.04 & $0.42(0.18-0.98)$ \\
\hline
\end{tabular}

rs11549465 polymorphism: comparison of general clinical data in patients with different genotypes

Compared with patients with the CC genotype, patients with the CT allele showed lower $\mathrm{SCr}(\mathrm{P}<0.05)$, and there was no significant difference between the 2 groups in regard to gender, age, blood glucose, body mass index, $C$ peptide, TG, and other indicators $(P>0.05$; Table 4$)$.

\section{Logistic regression analysis of DN-related factors}

Using the incidence of DN in patients as the categorical variable, gender, age, blood pressure, body mass index, fasting plasma glucose, 2-h postprandial plasma glucose, fasting 
C-peptide, PCP, TG, total cholesterol, low-density lipoprotein cholesterol, HDL-C, HbA1C, and rs 11549465 genotypes were considered independent variables for logistic regression analysis. The results showed that high $\mathrm{HbA} 1 \mathrm{c}$ and low HDL-C were risk factors for DN; the T allele and constants were excluded in the equation $(P>0.05$, Table 5$)$.

Table 4. Comparison of clinical data in patients with different genotypes.

\begin{tabular}{lccc}
\hline & CC $(\mathrm{N}=218)$ & CT $(\mathrm{N}=26)$ & P value \\
\hline Gender $($ male/female $)$ & $118 / 100$ & $15 / 11$ & 0.60 \\
Age (years) & $54.64 \pm 15.14$ & $55.19 \pm 12.02$ & 0.86 \\
SBP $(\mathrm{mmHg})$ & $133.52 \pm 19.37$ & $137.7 \pm 17.84$ & 0.30 \\
DBP $(\mathrm{mmHg})$ & $82.60 \pm 14.35$ & $84.23 \pm 14.29$ & 0.59 \\
BMI $\left(\mathrm{kg} / \mathrm{m}^{2}\right)$ & $23.49 \pm 3.31$ & $23.70 \pm 2.60$ & 0.73 \\
FPG $(\mathrm{mM})$ & $8.83 \pm 3.90$ & $9.12 \pm 3.40$ & 0.71 \\
2hPG $(\mathrm{mM})$ & $15.26 \pm 7.93$ & $15.67 \pm 5.02$ & 0.80 \\
FCP $(\mathrm{ng} / \mathrm{mL})$ & $1.46 \pm 1.21$ & $1.57 \pm 1.56$ & 0.68 \\
2hCP $(\mathrm{mg} / \mathrm{mL})$ & $3.67 \pm 3.03$ & $4.07 \pm 3.89$ & 0.54 \\
TG $(\mathrm{mM})$ & $1.35(0.91,2.07)$ & $1.54(1.07,2.44)$ & 0.32 \\
TC $(\mathrm{mM})$ & $4.75 \pm 1.50$ & $4.82 \pm 2.22$ & \\
LDL-C $(\mathrm{mM})$ & $3.01 \pm 1.31$ & $2.7 \pm 1.00$ & \\
HDL-C $(\mathrm{mM})$ & $1.17 \pm 0.52$ & $1.03 \pm 0.45$ & \\
HbA1 $/ \%$ & $10.4 \pm 7.1$ & $9.90 \pm 2.50$ & \\
BUN $(\mathrm{mM})$ & $8.14 \pm 7.06$ & $7.17 \pm 5.82$ & 0.83 \\
SCr $(\mu \mathrm{M})$ & $131.1 \pm 62.10$ & $102.20 \pm 55.21$ & 0.20 \\
\hline
\end{tabular}

Table 5. Logistic regression analysis of DN-related factors.

\begin{tabular}{lccccc}
\hline Categorical variable & Independent variables & $\beta$ & OR & OR $(95 \% \mathrm{Cl})$ & $\mathrm{P}$ value \\
\hline DN & HbA1c & 0.13 & 0.88 & $0.98-0.78$ \\
& HDL-C & -0.79 & 2.21 & $4.08-1.19$ & 0.02 \\
\hline
\end{tabular}

\section{DISCUSSION}

There are multiple-single nucleotide polymorphism sites in the exons of the HIF-1a gene. The Pro582Ser polymorphism can improve the expression of HIF-1 $\alpha$ during hypoxia and high glucose stress in cells (Gu et al., 2013). In the early development of DN, the presence of hypoxia can be detected in the renal outer medulla of DM animal models. Tubulointerstitial chronic hypoxia may be a common pathway of glomerular disease progressing to end-stage renal disease (Nangaku, 2006), suggesting that hypoxia may be a major causative factor of DN (Palm et al., 2011). Expression of HIF-1 in the kidney of DN rats was regulated compared with normal kidney, suggesting that HIF-1 is involved in DN pathogenesis (Li et al., 2011). Changes in C $\rightarrow$ T in the HIF-1a rs 11549465 polymorphism transformed proline into serine (Pro582Ser); in the absence of oxygen and hyperglycemia, the level of transcription of the CT and TT genotypes increased compared with the CC genotype, suggesting that the polymorphism may affect the expression of HIF-1 $\alpha$, altering its regulation of downstream genes. Thus, it is involved in the pathogenesis of DN (Gu et al., 2013).

In the present study, we examined HIF-1 $\alpha$ gene polymorphisms in type 2 diabetic patients with or without DN to further confirm the rs 11549465 polymorphism in HIF-1 $\alpha$ exons. Compared with the DN group, the T allele carrying rate of the HIF-1a rs 11549465 polymorphism in the DM group was higher, suggesting that the T allele may be a protective factor for DN. Logistic regression analysis showed that the gene polymorphism was excluded in the equation, which may be related to the following factors. In ischemia and hypoxia, the body will undergo a compensatory 
response to increase the expression of HIF-1 $\alpha$ and its downstream target genes (such as heme oxygenase-1 and glycolytic enzymes) in order for cells to adapt to hypoxia; this adjustment reflects the protective effect of HIF in the kidneys (Zhang et al., 2007), making the kidneys more adaptive to a hypoxic environment and may delay the progression of chronic kidney disease (Tanaka et al., 2005). However, the high expression of HIF-1a was also a harmful factor for kidney fibrosis and hypoxia. Under chronic kidney hypoxia conditions, high levels of HIF can activate its downstream target genes, such as endothelin-1 and vascular endothelial growth factor. In addition, in diabetic nephropathy, increased angiotensin II causes the long-term contraction of renal blood vessels, leading to increased ischemia and hypoxia of renal tissues, further promoting the expression of HIF. Vascular endothelial growth factor, endothelin-1, and angiotensin II are important proinflammatory and fibrogenic factors (Seccia et al., 2008; Lin et al., 2011), and there is a synergistic effect among them in promoting renal fibrosis. In the pathogenesis of DN, high expression of HIF-1a enhances the tolerance of kidney tissues and cells to hypoxia, playing a protective role in the kidneys. However, HIF also induces kidney fibrosis and kidney damage. Therefore, the HIF-1a polymorphism Pro582Ser may play different an opposite role in the development of DN at different stages. Early in DN, upregulation of HIF-1 $\alpha$ may delay further deterioration of the disease by improving adaptability to the hypoxic environment. When the progression of DN reaches a specific stage, extensive microvascular disease and microcirculation obstacles appeared; meanwhile, decompensation occurred in the regulation of hypoxia by HIF-1 $\alpha$, so that its increase accelerated interstitial fibrosis and disease progression.

In this study, multivariate analysis showed that HIF-1a gene Pro582Ser polymorphism may have a protective effect against DN, indicating that precise regulation of HIF-1 1 a has important implications for the development of DN. Our results should be further examined in multi-stage studies with large sample sizes.

\section{Conflicts of interest}

The authors declare no conflict of interest.

\section{ACKNOWLEDGMENTS}

Research supported by the Natural Science Key Foundation of the Anhui Higher Education Institutions, China (Grant \#KJ2014A156).

\section{REFERENCES}

Gu HF, Zheng X, Abu Seman N, Gu T, et al. (2013). Impact of the hypoxia-inducible factor-1 $\alpha$ (HIF1A) Pro582Ser polymorphism on diabetes nephropathy. Diabetes Care 36: 415-421.

Li J, Wang QL, Sun X, et al. (2011). Hypoxia-inducible factor-1 expression levels in rat kidney of diabetic nephropathy. Chin. J. Gerontol. 31: 4394-4396.

Lin SL, Chang FC, Schrimpf C, Chen YT, et al. (2011). Targeting endothelium-pericyte cross talk by inhibiting VEGF receptor signaling attenuates kidney microvascular rarefaction and fibrosis. Am. J. Pathol. 178: 911-923.

Nangaku M (2006). Chronic hypoxia and tubulointerstitial injury: a final common pathway to end-stage renal failure. J. Am. Soc. Nephrol. 17: 17-25.

Palm F, Nordquist L, Wilcox CS and Hansell P (2011). Oxidative stress and hypoxia in the pathogenesis of diabetic nephropathy In: Studies on Renal Disorders. Humana Press, New York, 559-586.

Seccia TM, Maniero C, Belloni AS, Guidolin D, et al. (2008). Role of angiotensin II, endothelin-1 and L-type calcium channel in the development of glomerular, tubulointerstitial and perivascular fibrosis. J. Hypertens. 26: 2022-2029. 
Semenza GL (2010). Oxygen homeostasis. Wiley Interdiscip. Rev. Syst. Biol. Med. 2: 336-361.

Tanaka T and Nangaku M (2010). The role of hypoxia, increased oxygen consumption, and hypoxia-inducible factor- 1 alpha in progression of chronic kidney disease. Curr. Opin. Nephrol. Hypertens. 19: 43-50.

Tanaka T, Kojima I, Ohse T, Ingelfinger JR, et al. (2005). Cobalt promotes angiogenesis via hypoxia-inducible factor and protects tubulointerstitium in the remnant kidney model. Lab. Invest. 85: 1292-1307.

Zhang XL, Liu H, Zou JZ, et al. (2007). Hypoxia inducible factor $1 \alpha$ activation attenuates ischemia reperfusion injury of the kidney in mice. Chin. J. Nephrol. 23: 448-452. 